\title{
Characterizations of regularities in ordered semihyper- groups in terms of some generalized union soft hyperideals
}

\author{
Jirapong Mekwian, Somsak Lekkoksung, Nareupanat Lekkoksung*
}

Division of Mathematics, Faculty of Engineering, Rajamangala University of Technology Isan, Khon Kaen Campus, Khon Kaen 40000, Thailand.

\begin{abstract}
A soft set of $E$ over $U$ is a mapping from $E$ to the set of all subsets of $U$. There are many studies that apply the concept of soft sets to investigate the properties of some algebraic structures. The notions of $(M, N)$-union soft left (resp., right) hyperideals in ordered semihypergroups were introduced by Farooq, Khalaf, and Khan. These concepts are generalizations of uni-soft left and right hyperideals. Ordered semihypergroups can be characterized by many mathematical concepts, such as their hyperideals, fuzzy hyperideals, and soft hyperideals. In this paper, we apply the notions of ( $M, N$ )-union soft left (resp., right) hyperideals to characterize some regularities of ordered semihypergroups: regular, weakly regular, and intra-regular ordered semihypergroups.
\end{abstract}

Keywords: Ordered semihypergroup, $(M, N)$-union soft left hyperideal, $(M, N)$-union soft right hyperideal, regular, weakly regular, intra-regular.

2020 MSC: 20N20.

(C)2022 All rights reserved.

\section{Introduction}

Hyperstructure theory was first introduced by Marty [24]. The theory is widely studied from the theoretical point of view and for its applications to many areas of pure and applied properties (see $[3,4,5])$.

The idea of semihypergroups (also called hypersemigroup or multisemigroup) was first investigated by Bonansinga, and Corsini [2,4]. Later studied by many authors, for example by Mahmood [23], Hila et al. [11], Davvaz et al. [5], Gu and Tang [9], Heidari and Davvaz [10], and many others. The concept of ordered semihypergroups is a generalization of the concept of ordered semigroups. Indeed, every ordered semigroup can be regarded as an ordered semihypergroup (see [14]).

Recently, many interesting properties of ordered semihypergroups were investigated by many scientists. In 2021, Gu [8] extended classification of weakly semiprime segments in ordered semigroups to ordered semihypergroups. By this similar idea, extending a study in ordered semigroups to ordered

\footnotetext{
*Corresponding author

Email address: nareupanat.le@rmuti.ac.th (Nareupanat Lekkoksung)

doi: $10.22436 /$ jmcs.026.03.08
}

Received: 2021-07-14 Revised: 2021-03-10 Accepted: 2021-08-19 
semihypergroups, Yiarayong et al. [29] classified completely prime ideals in ordered semihypergroups. By these two studies, the primness properties of hyperideals are spotted considered.

We mention that some classical algebraic structures are now extended and widely investigated. For example, in 2013, the authors [27] considered a generalization of semihypergroups which is called a left almost semihypergroup. The left almost concept was applied to polygroups in [28]. The concept of unisoft structure was applied to ordered $\Gamma$-semihypergroups by Khan et al. [19]. The notion of ordered *-semihypergroups was studied in 2021 by Tang and Yaqoob [26].

Molodtsov [25] introduced the concept of soft sets as a new mathematical tool for dealing with uncertainties that are free from the difficulties that have troubled the usual theoretical approaches. Anvariyeh et al. [1] initiated soft semihypergroups by using the soft set theory. They introduced soft semihypergroups, soft subsemihypergroups, soft hyperideals and studied several related properties. Naz and Shabir [22] applied soft set theory to semihypergroups.

The concept of union-soft semigroups, union-soft l-ideals, union-soft r-ideals, and union-soft semiprime soft sets have been considered by Jun et al. [13]. As a generalization of the concept of soft semigroups, soft left (right) ideals and left (right) idealistic soft semigroups, in 2010, Jun et al. [12] initiated the study of soft ordered semigroups, soft left (right) ideals, and left (right) idealistic soft ordered semigroups. Shabir et al. [20] introduced the concept of union-soft version of semiprime quasi-ideals of ordered semigroups and characterized completely regular ordered semigroup in terms of semiprime union-soft quasi-ideals. Khan et al. [21] characterized weakly regular, intra-regular, and semisimple ordered semigroups by the properties of their union-soft ideals.

This present paper is to study ordered semihypergroups in the context of union soft left (right) hyperideals. The notion of generalized union soft left (right) hyperideals is studied, and their related properties are discussed. We characterize regular and weakly regular ordered semihypergroups in terms of generalized union soft left (right) hyperideals. Finally, we also characterize intra-regular using union soft hyperideals.

\section{Preliminaries}

This section will recall the basic terms and definitions from the hyperstructure theory and the soft set theory.

Definition 2.1 ([5]). A map $\circ: \mathrm{H} \times \mathrm{H} \rightarrow \mathcal{P}^{*}(\mathrm{H})$ is called a hyperoperation on the set $\mathrm{H}$, where $\mathrm{H}$ is a nonempty set and $\mathcal{P}^{*}(\mathrm{H})$ denotes the set of all nonempty subsets of $\mathrm{H}$.

A hypergroupoid is a structure $\langle\mathrm{H} ; 0\rangle$ comprising of a nonempty set $\mathrm{H}$ with together a (binary) hyperoperation on $\mathrm{H}$.

Let $A$ and $B$ be two nonempty subsets of $H$. We define

$$
A \circ B=\bigcup_{a \in A, b \in B} a \circ b
$$

Definition 2.2 ([5]). A hypergroupoid $\langle\mathrm{H} ; 0\rangle$ satisfying

$$
x \circ(y \circ z)=(x \circ y) \circ z
$$

for all $x, y, z \in H$ is called a semihypergroup.

Definition 2.3 ([5]). The hyperstructure $\langle\mathrm{H} ; 0, \leqslant\rangle$ is called an ordered semihypergroup if

1. $\langle\mathrm{H} ; 0\rangle$ is a semihypergroup;

2. $\langle\mathrm{H} ; \leqslant\rangle$ is a partially ordered set;

3. for every $a, b, c \in H$ if $a \leqslant b$, then $a \circ c \leqslant b \circ c$ and $c \circ a \leqslant c \circ b$. Here, $A \leqslant B$ means that for each $a \in A$ there exists $b \in B$ such that $a \leqslant b$. 
For simplicity, we denoted an ordered semihypergroup $\langle\mathrm{H} ; 0, \leqslant\rangle$ by its carrier set as a bold letter $\mathbf{H}$. Let $\mathbf{H}$ be an ordered semihypergroup. For $\mathrm{K} \subseteq \mathrm{H}$, we denote

$$
(\mathrm{K}]:=\{\mathrm{a} \in \mathrm{H}: \mathrm{a} \leqslant \mathrm{k} \text { for some } \mathrm{k} \in \mathrm{K}\} .
$$

A nonempty subset $A$ of $H$ is called a subsemihypergroup of $\mathbf{H}$ if $\langle A ; 0, \leqslant\rangle$ is an ordered semihypergroup.

Definition 2.4 ([5]). Let $\mathbf{H}$ be an ordered semihypergroup. A nonempty subset $\mathrm{R}$ of $\mathrm{H}$ is called a right hyperideal of $\mathbf{H}$ if

1. $\mathrm{R} \circ \mathrm{H} \subseteq \mathrm{R}$;

2. for $a \in H$ and $b \in R$, if $a \leqslant b$, then $a \in R$.

Definition 2.5 ([5]). Let $\mathbf{H}$ be an ordered semihypergroup. A nonempty subset $\mathrm{L}$ of $\mathrm{H}$ is called a left hyperideal of $\mathbf{H}$ if

1. $\mathrm{H} \circ \mathrm{L} \subseteq \mathrm{L}$;

2. for $a \in H$ and $b \in L$, if $a \leqslant b$, then $a \in L$.

A nonempty subset I of $\mathrm{H}$ is called a hyperideal of $\mathbf{H}$ if it is both a left hyperideal and a right hyperideal of $\mathbf{H}$.

For any $a \in H$, we denote by $R(a)$ and $L(a)$ the right hyperideal of $\mathbf{H}$ generated by $\mathbf{a}$ and left hyperideal of $\mathbf{H}$ generated by $\mathbf{a}$, respectively. One can prove that $R(a)=(a \cup(a \circ H)]$ and $L(a)=$ $(a \cup(H \circ a)]$.

From now on, $U$ is an initial universe set, $E$ is a set of parameters, $\mathcal{P}(U)$ is the set of all subsets of $U$ and $A, B, C, \cdots$ are nonempty subsets of $E$.

Definition 2.6 ([25]). A soft set $f_{A}$ of $E$ over $U$ is defined as $f_{A}: E \rightarrow \mathcal{P}(U)$ such that $f_{A}(x):=\emptyset$ if $x \notin A$.

Hence, $f_{A}$ is also called an approximation function. A soft set $f_{A}$ of $E$ over $U$ can be represented by the set of ordered pairs

$$
f_{A}:=\left\{\left(x, f_{A}(x)\right): x \in E \text { and } f_{A}(x) \in \mathcal{P}(U)\right\} .
$$

By the above definition, a soft set is a parameterized family of subsets of $\mathrm{U}$. From now on, we denote the set of all soft sets of $E$ over $U$ by $S_{E}(U)$.

Let $A \subseteq E$. The characteristic soft sets $\chi_{A}$ and $\chi_{A}^{c}$ of $E$ over $U$ are defined by

$$
\chi_{A}(x):= \begin{cases}u & \text { if } x \in A, \\ \emptyset & \text { otherwise }\end{cases}
$$

and

$$
\chi_{A}^{c}(x):= \begin{cases}\emptyset & \text { if } x \in A, \\ U & \text { otherwise, }\end{cases}
$$

for all $x \in E$ (see [18]). We observe that, for any $A \subseteq E$, we have $\chi_{A}^{c}(x)=\chi_{E \backslash A}(x)$ for all $x \in E$.

Let $f_{A}, f_{B} \in S_{E}(U)$. In [25], Molodtsov defined binary relations and operations on $S_{E}(U)$ as follows.

1. The soft set $f_{A}$ is said to be a soft subset of $f_{B}$, denote by $f_{A} \sqsubseteq f_{B}$, if $f_{A}(x) \subseteq f_{B}(x)$ for all $x \in E$. Note that we also write $f_{B} \sqsupseteq f_{A}$ instead of $f_{A} \sqsubseteq f_{B}$.

2. The soft sets $f_{A}$ and $f_{B}$ are said to be equal, denoted by $f_{A}=f_{B}$, if $f_{A} \sqsubseteq f_{B}$ and $f_{B} \sqsubseteq f_{A}$.

3. The soft union of $f_{A}$ and $f_{B}$, denoted by $f_{A} \sqcup f_{B}=f_{A \cup B}$, is defined by $\left(f_{A} \sqcup f_{B}\right)(x):=f_{A}(x) \cup f_{B}(x)$ for all $x \in E$. 
4. The soft intersection of $f_{A}$ and $f_{B}$, denoted by $f_{A} \sqcap f_{B}=f_{A \cap B}$, is defined by

$$
\left(f_{A} \sqcap f_{B}\right)(x):=f_{A}(x) \cap f_{B}(x),
$$

for all $x \in E$.

Khan et al. [18] apply the concept of soft sets to ordered semihypergroups. Let $\mathbf{H}$ be an ordered semihypergroup. We call any soft set of $\mathrm{H}$ over $\mathrm{U}$ by a soft set of $\mathbf{H}$ over $\mathrm{U}$. Denote the set of all soft sets of $\mathbf{H}$ over $\mathrm{U}$ by $\mathbf{H}(\mathrm{U})$. In this case, $\mathrm{S}_{\mathrm{H}}(\mathrm{U})=\mathbf{H}(\mathrm{U})$.

Let $\mathbf{H}$ be an ordered semihypergroup and $a \in H$. Then, we define

$$
\mathbf{H}_{\mathrm{a}}:=\{(x, y) \in \mathrm{H} \times \mathrm{H}: \mathbf{a} \leqslant x \circ y \text { for some } x, y \in H\} .
$$

For any $f_{A}, f_{B} \in H(U)$, define a new soft set $f_{A} \diamond f_{B}$ by

$$
\left(f_{A} \diamond f_{B}\right)(a)= \begin{cases}\bigcap_{(x, y) \in \mathbf{H}_{a}}\left(f_{A}(x) \cup f_{B}(y)\right) & \text { if } \mathbf{H}_{a} \neq \emptyset, \\ u & \text { otherwise, }\end{cases}
$$

for all $a \in H$.

Besides, they defined the notions of union soft subsemihypergroups and union soft left (resp., right) hyperideals of ordered semihypergroups. The related properties were investigated. This paper focuses on a generalized concept of union soft left (resp., right) hyperideals of ordered semihypergroups, so-called $(\mathrm{M}, \mathrm{N})$-union soft left (resp., right) hyperideals which were introduced by Farooq et al. [7].

Now, we always assume that $\emptyset \subseteq M \subset \mathrm{N} \subseteq \mathrm{U}$. We recall some knowledge concerning the concept of $(M, N)$-union soft left (resp., right) hyperideals. Let $\mathbf{H}$ be an ordered semihypergroup. For any $f_{A}, f_{B} \in$ $\mathbf{H}(\mathrm{U})$, we define a relation $\beth_{[M, N]}$ by

$$
f_{A} \sqsupseteq_{[M, N]} f_{B} \quad \text { if and only if } \quad\left(f_{A}(x) \cup M\right) \cap N \supseteq\left(f_{B}(x) \cup M\right) \cap N,
$$

for all $x \in H$. In particular, $f_{A}=[M, N] f_{B}$ if $f_{A} \sqsupseteq_{[M, N]} f_{B}$ and $f_{B} \sqsupseteq_{[M, N]} f_{A}$.

By this setting, they obtained the following theorem.

Theorem 2.7 ([7, Theorem 4.1]). Let $\mathbf{H}$ be an ordered semihypergroup. Then $\left\langle\mathbf{H}(\mathrm{U}) ; \diamond, \beth_{[M, N]}\right\rangle$ is an ordered semihypergroup.

Definition 2.8 ([7]). Let $\mathbf{H}$ be an ordered semihypergroup. A soft set $f_{A}$ of $\mathbf{H}$ over $U$ is called an $(M, N)$ union soft subsemihypergroup of $\mathbf{H}$ over $\mathrm{U}$ if

$$
\left(\bigcup_{a \in x \circ y} f_{A}(a)\right) \cap N \subseteq f_{A}(x) \cup f_{A}(y) \cup M,
$$

for all $x, y \in H$.

Definition 2.9 ([7]). Let $\mathbf{H}$ be an ordered semihypergroup. A soft set $f_{A}$ of $\mathbf{H}$ over $U$ satisfying $x \leqslant y$ implies $f_{A}(x) \cap N \subseteq f_{A}(y) \cup M$ is called

1. an $(M, N)$-union soft left hyperideal of $\mathbf{H}$ over $U$ if $\left(\bigcup_{a \in x \circ y} f_{A}(a)\right) \cap N \subseteq f_{A}(y) \cup M$;

2. an $(M, N)$-union soft right hyperideal of $\mathbf{H}$ over $U$ if $\left(\bigcup_{a \in x \circ y} f_{A}(a)\right) \cap N \subseteq f_{A}(x) \cup M$.

A soft set of $\mathbf{H}$ over $\mathrm{U}$ is called an $(\mathrm{M}, \mathrm{N})$-union soft hyperideal of $\mathbf{H}$ over $\mathrm{U}$ if it is both an $(\mathrm{M}, \mathrm{N})$ uni-soft left hyperideal and an $(\mathrm{M}, \mathrm{N})$-uni-soft right hyperideal of $\mathbf{H}$ over $\mathrm{U}$.

By the above definitions given by Farooq et al., we can see that the concept of $(M, N)$-union soft left (resp., right) hyperideals is a generalization of union soft left (resp., right) hyperideals provided by Khan et al. in [17]. That is, an ( $M, N)$-union soft left (resp., right) hyperideal is a union soft left (resp., right) hyperideal whenever $\mathrm{M}=\emptyset$ and $\mathrm{N}=\mathrm{U}$. 
Example 2.10. Let $H=\{a, b, c\}$. Define the hyperoperation $\circ$ on $H$ by the following table:

\begin{tabular}{c|ccc}
$\circ$ & $a$ & $b$ & $c$ \\
\hline$a$ & $\{a\}$ & $\{a\}$ & $\{a\}$ \\
$b$ & $\{a\}$ & $\{a\}$ & $\{a\}$ \\
$c$ & $\{a\}$ & $\{a, b\}$ & $\{c\}$
\end{tabular}

Define a partial order $\leqslant$ on $H$ as follows: $\leqslant:=\Delta_{H} \cup\{(a, b)\}$, where $\Delta_{H}=\{(x, x): x \in H\}$. Then $\mathbf{H}:=$ $\langle H ; \circ, \leqslant\rangle$ is an ordered semihypergroup. Let $U=\{1,2,3,4,5,6,7\}$ be a set. Define $f_{H}: H \rightarrow \mathcal{P}(U)$ by

$$
f_{H}(x)= \begin{cases}\{3,4,5,6\} & \text { if } x=a, \\ \{1,2,3\} & \text { if } x=b, \\ \{3,4,5,6,7\} & \text { if } x=c,\end{cases}
$$

for all $x \in H$. We put $M=\{4,5\}$ and $N=\{2,3,4,5\}$. Then, we can carefully calculate that $f_{H}$ is an $(M, N)$-union soft left hyperideal of $\mathbf{H}$ over $U$. In contrast, $f_{H}$ is not an $(M, N)$-union soft right hyperideal of $\mathbf{H}$ over $\mathrm{U}$ since there exists $\mathrm{b} \in \mathrm{c} \circ \mathrm{b}$ such that $\mathrm{f}_{\mathrm{H}}(\mathrm{b}) \cap \mathrm{N}=\{2,3\} \nsubseteq\{3,4,5,6,7\}=\mathrm{f}_{\mathrm{H}}(\mathrm{c}) \cup M$.

Example 2.11. Let $H=\{a, b, c\}$. Define the hyperoperation $\circ$ on $H$ by the following table:

\begin{tabular}{c|ccc}
$\circ$ & $\mathrm{a}$ & $\mathrm{b}$ & $\mathrm{c}$ \\
\hline $\mathrm{a}$ & $\{\mathrm{a}\}$ & $\{\mathrm{a}\}$ & $\{\mathrm{a}\}$ \\
$\mathrm{b}$ & $\{\mathrm{a}\}$ & $\{\mathrm{a}\}$ & $\{\mathrm{a}\}$ \\
$\mathrm{c}$ & $\mathrm{H}$ & $\mathrm{H}$ & $\mathrm{H}$
\end{tabular}

Define a partial order $\leqslant$ on $H$ as follows: $\leqslant:=\Delta_{H} \cup\{(a, c),(b, c)\}$, where $\Delta_{H}=\{(x, x): x \in H\}$. Then $\mathbf{H}:=\langle\mathrm{H} ; \circ, \leqslant\rangle$ is an ordered semihypergroup. Let $\mathrm{U}=\mathbb{N}$ be a set. Define $\mathrm{f}_{\mathrm{H}}: \mathrm{H} \rightarrow \mathcal{P}(\mathrm{U})$ by

$$
f_{H}(x)= \begin{cases}2 \mathbb{N} & \text { if } x=a, b, \\ \{1,2,3\} & \text { if } x=c,\end{cases}
$$

for all $x \in H$. We put $M=\{4,5\}$ and $N=\{2,3,4,5\}$. Then, we can carefully calculate that $f_{H}$ is an $(M, N)$-union soft right hyperideal of $\mathbf{H}$ over $\mathrm{U}$. But, $f_{H}$ is not an $(M, N)$-union soft left hyperideal of $\mathbf{H}$ over $U$ since there exists $c \in a \circ$ a such that $f_{H}(c) \cap N=\{2,3\} \nsubseteq 2 \mathbb{N} \cup\{5\}=f_{H}(a) \cup M$.

\section{Main results}

In this main results section, we introduce new soft sets and use them to characterize some particular classes of ordered semihypergroups: regular, intra-regular, and weakly-regular.

Before starting our main theorems, we provide a characterization of $(M, N)$-union soft subsemihypergroups in terms of the operation $\diamond$.

Theorem 3.1. Let $\mathbf{H}$ be an ordered semihypergroup, and $\mathrm{f}_{\mathrm{A}}$ be a soft set of $\mathbf{H}$ over $\mathrm{U}$ such that $\mathrm{x} \leqslant \mathrm{y}$ implies $\mathrm{f}_{\mathrm{A}}(\mathrm{x}) \cap \mathrm{N} \subseteq \mathrm{f}_{\mathrm{A}}(\mathrm{y}) \cup \mathrm{M}$ for any $\mathrm{x}, \mathrm{y} \in \mathrm{H}$. Then the following statements are equivalent:

1. $f_{A}$ is an $(M, N)$-union soft subsemihypergroup of $\mathbf{H}$ over $\mathrm{U}$;

2. $f_{A} \diamond f_{A} \sqsupseteq_{[M, N]} f_{A}$.

Proof. (1) $\Rightarrow(2)$. Let $x \in H$. Since $M \supseteq\left[f_{A}(x) \cup M\right] \cap N$, we obtain that

$$
\left[\left(f_{A} \diamond f_{A}\right)(x) \cup M\right] \cap N=M \supseteq\left[f_{A}(x) \cup M\right] \cap N,
$$

whenever $\mathbf{H}_{x}=\emptyset$. We suppose that $\mathbf{H}_{x} \neq \emptyset$. Let $(u, v) \in \mathbf{H}_{x}$. Then $x \leqslant t$ for some $t \in \mathfrak{u} \circ v$. By our presumption, we have that

$$
f_{A}(x) \cap N \subseteq f_{A}(t) \cup M \subseteq \bigcup_{t \in u o v}\left(f_{A}(t) \cup M\right)
$$


This means that $f_{A}(x) \cap N \subseteq \bigcup_{t \in \mathcal{u} o v}\left(f_{A}(t) \cup M\right)$ for any $(u, v) \in \mathbf{H}_{x}$. Therefore,

$$
\begin{aligned}
{\left[\left(f_{A} \diamond f_{A}\right)(x) \cup M\right] \cap N } & =\left[\left(\bigcap_{(u, v) \in \mathbf{H}_{x}}\left(f_{A}(u) \cup f_{A}(v)\right)\right) \cup M\right] \cap N \\
& =\left[\left(\bigcap_{(u, v) \in \mathbf{H}_{x}}\left(f_{A}(u) \cup f_{A}(v)\right)\right) \cup M \cup M\right] \cap N \\
& =\left[\left(\bigcap_{(u, v) \in \mathbf{H}_{x}}\left(f_{A}(u) \cup f_{A}(v) \cup M\right)\right) \cup M\right] \cap N \\
& \supseteq\left[\left(\bigcap_{(u, v) \in \mathbf{H}_{x}}\left(\bigcup_{c \in u o v} f_{A}(c) \cap N\right)\right) \cup M\right] \cap N \\
& \supseteq\left[\left(\bigcap_{(u, v) \in \mathbf{H}_{x}}\left(f_{A}(x) \cap N\right)\right) \cup M\right] \cap N \\
& =\left(\bigcap_{(u, v) \in \mathbf{H}_{x}} f_{A}(x) \cup M\right) \cap N \\
& =\left(f_{A}(x) \cup M\right) \cap N .
\end{aligned}
$$

By these two cases, we obtain $f_{A} \diamond f_{A} \sqsupseteq_{[M, N]} f_{A}$.

$(2) \Rightarrow(1)$. Assume that $f_{A} \diamond f_{A} \sqsupseteq_{[M, N]} f_{A}$. That is, $\left(f_{A}(a) \cup M\right) \cap N \subseteq\left[\left(f_{A} \diamond f_{A}\right)(z) \cup M\right] \cap N$ for all $z \in \mathrm{H}$. Let $x, y \in H$. Then, for any $a \in x \circ y$, we obtain

$$
\begin{aligned}
f_{A}(a) \cap N & \subseteq\left(f_{A}(a) \cup M\right) \cap N \\
& \subseteq\left(\left[\left(f_{A} \diamond f_{A}\right)(a) \cup M\right] \cap N\right) \cap N \\
& \subseteq\left[\left(f_{A} \diamond f_{A}\right)(a) \cup M\right] \cap N \\
& =\left[\bigcap_{(b, c) \in \mathbf{H}_{a}}\left[f_{A}(b) \cup f_{A}(c)\right] \cup M\right] \cap N \\
& \subseteq\left[\bigcap_{(b, c) \in \mathbf{H}_{a}}\left[f_{A}(b) \cup f_{A}(c)\right] \cup M\right] \\
& \subseteq f_{A}(x) \cup f_{A}(y) \cup M .
\end{aligned}
$$

Thus, $\left(\cup_{a \in \text { xoy }} f_{A}(a)\right) \cap N \subseteq f_{A}(x) \cup f_{A}(y) \cup M$. Hence, $f_{A}$ is an $(M, N)$-union soft subsemihypergroup of $\mathbf{H}$ over $\mathrm{U}$.

In [7], the authors showed the following result.

Theorem 3.2 ([7, Theorem 4.6 and 4.7]). Let $\mathbf{H}$ be an ordered semihypergroup, and $\mathrm{f}_{\mathrm{A}}$ be a soft set of $\mathbf{H}$ over $\mathrm{U}$ such that $\mathrm{x} \leqslant \mathrm{y}$ implies $\mathrm{f}_{\mathrm{A}}(\mathrm{x}) \cap \mathrm{N} \subseteq \mathrm{f}_{\mathrm{A}}(\mathrm{y}) \cup \mathrm{M}$ for any $\mathrm{x}, \mathrm{y} \in \mathrm{H}$. Then the following statements are equivalent:

1. $\mathrm{f}_{\mathrm{A}}$ is an $(\mathrm{M}, \mathrm{N})$-union soft left (resp., right) hyperideal of $\mathbf{H}$ over $\mathrm{U}$;

2. $\chi_{H}^{C} \diamond f_{A} \sqsupseteq_{[M, N]} f_{A}\left(\right.$ resp., $\left.f_{A} \diamond \chi_{H}^{C} \sqsupseteq_{[M, N]} f_{A}\right)$.

By applying Theorem 3.2, one can prove the following corollary. 
Corollary 3.3. Let $\mathbf{H}$ be an ordered semihypergroup, and $\mathrm{f}_{\mathrm{A}}$ be a soft set of $\mathbf{H}$ over $\mathrm{U}$ such that $\mathrm{x} \leqslant \mathrm{y}$ implies $\mathrm{f}_{\mathrm{A}}(\mathrm{x}) \cap \mathrm{N} \subseteq \mathrm{f}_{\mathrm{A}}(\mathrm{y}) \cup \mathrm{M}$ for any $\mathrm{x}, \mathrm{y} \in \mathrm{H}$. Then the following statements are equivalent:

1. $f_{A}$ is an $(M, N)$-union soft hyperideal of $\mathbf{H}$ over $\mathrm{U}$;

2. $\chi_{H}^{c} \diamond f_{A} \sqsupseteq_{[M, N]} f_{A}$ and $f_{A} \diamond \chi_{H}^{c} \sqsupseteq_{[M, N]} f_{A}$.

Theorem 3.4. Let $\mathbf{H}$ be an ordered semihypergroup. If $\mathrm{f}_{\mathrm{A}}$ is an $(\mathrm{M}, \mathrm{N})$-union soft left (resp., right) hyperideal of $\mathbf{H}$ over $\mathrm{U}$, then $\mathrm{f}_{\mathrm{A}} \diamond \mathrm{f}_{\mathrm{A}} \sqsupseteq_{[\mathrm{M}, \mathrm{N}]} \mathrm{f}_{\mathrm{A}}$.

Proof. In this proof, we consider only the case that $f_{A}$ is an $(M, N)$-union soft left hyperideal of $\mathbf{H}$ over $U$. For the case that $f_{A}$ is an $(M, N)$-union soft right hyperideal of $\mathbf{H}$ over $U$, we can prove similarly. Let $x \in \mathrm{H}$. If $\mathbf{H}_{\mathrm{x}}=\emptyset$, then

$$
\left[\left(f_{A} \diamond f_{A}\right)(x) \cup M\right] \cap N=N \supseteq\left[f_{A}(x) \cup M\right] \cap N .
$$

Suppose that $\mathbf{H}_{x} \neq \emptyset$. Given $(u, v) \in \mathbf{H}_{x}$. Then $x \leqslant t$ for some $t \in u \circ v$. Since $f_{A}$ is an $(M, N)$-union soft left hyperideal of $\mathbf{H}$ over $\mathrm{U}$, we obtain that

$$
\begin{aligned}
f_{A}(x) \cap N & =\left(f_{A}(x) \cap N\right) \cap N \\
& \subseteq\left(f_{A}(t) \cup M\right) \cap N \\
& =\left(f_{A}(t) \cap N\right) \cup M \\
& \subseteq\left[\bigcup_{t \in u \circ v}\left(f_{A}(t) \cap N\right)\right] \cup M \\
& \subseteq\left(f_{A}(v) \cup M\right) \cup M \\
& \subseteq f_{A}(u) \cup f_{A}(v) \cup M .
\end{aligned}
$$

This illustrates that $f_{A}(u) \cup f_{A}(v) \cup M \supseteq f_{A}(x) \cap N$ for any $(u, v) \in \mathbf{H}_{x}$. Therefore,

$$
\begin{aligned}
{\left[\left(f_{A} \diamond f_{A}\right)(x) \cup M\right] \cap N } & =\left[\left(\bigcap_{(u, v) \in \mathbf{H}_{x}}\left(f_{A}(u) \cup f_{A}(v)\right)\right) \cup M\right] \cap N \\
& =\left[\left(\bigcap_{(u, v) \in \mathbf{H}_{x}}\left(f_{A}(u) \cup f_{A}(v) \cup M\right)\right) \cup M\right] \cap N \\
& \supseteq\left[\left(f_{A}(x) \cap N\right) \cup M\right] \cap N \\
& =\left(f_{A}(x) \cup M\right) \cap N .
\end{aligned}
$$

Altogether, we have that $f_{A} \diamond f_{A} \sqsupseteq_{[M, N]} f_{A}$.

Corollary 3.5. Let $\mathbf{H}$ be an ordered semihypergroup. If $\mathrm{f}_{\mathrm{A}}$ is an $(\mathrm{M}, \mathrm{N})$-union soft hyperideal of $\mathbf{H}$ over $\mathrm{U}$, then $f_{A} \diamond f_{A} \sqsupseteq[M, N] f_{A}$.

The following essential results were proved in [7].

Lemma 3.6 ([7, Theorem 4.4]). Let $\mathbf{H}$ be an ordered semihypergroup and A a nonempty subset of $\mathrm{H}$. Then the following conditions are equivalent:

1. $A$ is a left (resp., right) hyperideal of $\mathbf{H}$;

2. $\chi_{A}^{c}$ is an $(\mathrm{M}, \mathrm{N})$-union soft left (resp., right) hyperideal of $\mathbf{H}$ over $\mathrm{U}$.

Lemma 3.7 ([7, Corollary 4.1]). Let $\mathbf{H}$ be an ordered semihypergroup and $\mathrm{A}$ a nonempty subset of $\mathrm{H}$. Then the following conditions are equivalent: 
1. $A$ is a hyperideal of $\mathbf{H}$;

2. $\chi_{A}^{c}$ is an $(M, N)$-union soft hyperideal of $\mathbf{H}$ over $\mathrm{U}$.

To prove our main theorem, we need the following result.

Proposition 3.8. Let $\mathbf{H}$ be an ordered semihypergroup. Then the following conditions are hold:

1. $\chi_{A}^{\mathrm{c}} \sqcup \chi_{\mathrm{B}}^{\mathrm{c}}={ }_{[M, N]} \chi_{\mathrm{A} \cup \mathrm{B}}^{\mathrm{c}}$;

2. $\chi_{A}^{c} \diamond \chi_{B}^{c}={ }_{[M, N]} \chi_{(A \circ B}^{c}$.

Proof. In order to show that $\chi_{A}^{c} \sqcup \chi_{B}^{c}={ }_{[M, N]} \chi_{A \cup B}^{c}$, we let $x \in H$. If $x \in A \cup B$, then

$$
\left(\chi_{A}^{c} \sqcup \chi_{B}^{c}\right)(x)=\chi_{A}^{c}(x) \cup \chi_{B}^{c}(x)=\emptyset=\left(\chi_{A}^{c} \cup B\right)(x) .
$$

This implies that $\left[\left(\chi_{A}^{c} \sqcup \chi_{B}^{c}\right)(x) \cup M\right] \cap N=\left[\left(\chi_{A \cup B}^{c}\right)(x) \cup M\right] \cap N$. If $x \notin(A \cup B)$, then

$$
\left(\chi_{A}^{c} \sqcup \chi_{B}^{c}\right)(x)=\chi_{A}^{c}(x) \cup \chi_{B}^{c}(x)=U=\left(\chi_{A \cup B}^{c}\right)(x) .
$$

This implies that $\left[\left(\chi_{A}^{c} \sqcup \chi_{B}^{c}\right)(x) \cup M\right] \cap N=\left[\left(\chi_{A \cup B}^{c}\right)(x) \cup M\right] \cap N$. Hence, $\chi_{A}^{c} \sqcup \chi_{B}^{c}={ }_{[M, N]} \chi_{A \cup B}^{c}$.

Now, we show that $\chi_{A}^{c} \diamond \chi_{B}^{c}={ }_{[M, N]} \chi_{(A \circ B]}^{c}$. Let $x \in H$. If $x \in(A \circ B]$, then $\chi_{(A \circ B]}^{c}(x)=\emptyset$ and there exist $a \in A$ and $b \in B$ such that $x \leqslant a \circ b$. That is, $\mathbf{H}_{x} \neq \emptyset$ and $(a, b) \in \mathbf{H}_{x}$. Then

$$
\left(\chi_{A}^{c} \diamond \chi_{B}^{c}\right)(x)=\bigcap_{(u, v) \in \mathbf{H}_{x}}\left(\chi_{A}^{c}(u) \cup \chi_{B}^{c}(v)\right) \subseteq \chi_{A}^{c}(a) \cup \chi_{B}^{c}(b)=\emptyset .
$$

This means that $\left(\chi_{A}^{c} \diamond \chi_{B}^{c}\right)(x)=\emptyset$. Thus, we obtain that

$$
\left[\left(\chi_{A}^{c} \diamond \chi_{B}^{c}\right)(x) \cup M\right] \cap N=\left[\left(\chi_{(A \circ B]}^{c}\right)(x) \cup M\right] \cap N .
$$

Now, we suppose that $x \notin(A \circ B]$. Clearly, $\chi_{(A \circ B]}^{c}(x)=U$. If $\mathbf{H}_{x}=\emptyset$, then $\left(\chi_{A}^{c} \diamond \chi_{B}^{c}\right)(x)=U$. If $\mathbf{H}_{x} \neq \emptyset$, then $\left(\chi_{A}^{c} \diamond \chi_{B}^{c}\right)(x)=\bigcap_{(u, v) \in \mathbf{H}_{x}}\left(\chi_{A}^{c}(u) \cup \chi_{B}^{c}(v)\right)$. We show that $\chi_{A}^{c}(u) \cup \chi_{B}^{c}(v)=u$ for all $(u, v) \in \mathbf{H}_{x}$. Let $(a, b) \in \mathbf{H}_{x}$. Then, we have that $a \notin A$ or $b \notin B$. Otherwise, $x \leqslant a \circ b \in A \circ B$, which implies that $x \in(A \circ B]$. This is a contradiction. Therefore, $\chi_{A}^{c}(u) \cup \chi_{B}^{c}(v)=u$ for all $(u, v) \in \mathbf{H}_{x}$. That is, $\left(\chi_{A}^{c} \diamond \chi_{B}^{c}\right)(x)=U$. Altogether, we have $\left(\chi_{A}^{c} \diamond \chi_{B}^{c}\right)(x)=U=\chi_{(A \circ B]}^{c}(x)$. This implies that

$$
\left[\left(\chi_{A}^{c} \diamond \chi_{B}^{c}\right)(x) \cup M\right] \cap N=\left[\left(\chi_{(A \circ B]}^{c}\right)(x) \cup M\right] \cap N .
$$

By these two cases; $x \in(A \circ B]$ and $x \notin(A \circ B]$, we have that $\chi_{A}^{c} \diamond \chi_{B}^{c}={ }_{[M, N]} \chi_{(A \circ B]}^{c}$.

An ordered semihypergroup $\mathbf{H}$ is regular [16] if, for each element $a \in H$, there exists an element $x \in H$ such that $a \leqslant a \circ x \circ$ a. Equivalent definition:

1. $A \subseteq(A \circ H \circ A]$ for all $A \subseteq H$;

2. $a \in(a \circ H \circ a]$ for all $a \in H$.

Kehayopulu characterized regular ordered semihypergroups by using the concepts of left hyperideals and right hyperideals as follows.

Lemma 3.9 ([16, Theorem 3.9]). Let $\mathbf{H}$ be an ordered semihypergroup. Then the following conditions are equivalent:

1. $\mathbf{H}$ is regular;

2. $\mathrm{R} \cap \mathrm{L}=(\mathrm{R} \circ \mathrm{L}]$ for every right hyperideal $\mathrm{R}$ and every left hyperideal $\mathrm{L}$ of $\mathbf{H}$. 
Our first goal is to characterize regular ordered semihypergroups in terms of $(M, N)$-union soft left hyperideals and $(M, N)$-union soft right hyperideals. To do that, we need the following lemma.

Lemma 3.10. Let $\mathbf{H}$ be an ordered semihypergroup. Suppose that $\mathrm{f}_{\mathrm{A}}$ and $\mathrm{f}_{\mathrm{B}}$ is an $(\mathrm{M}, \mathrm{N})$-union soft right hyperideal and an $(\mathrm{M}, \mathrm{N})$-union soft left hyperideal of $\mathbf{H}$ over $\mathrm{U}$, respectively. Then we have $\mathrm{f}_{\mathrm{A}} \diamond \mathrm{f}_{\mathrm{B}} \beth_{[\mathrm{M}, \mathrm{N}]} \mathrm{f}_{\mathrm{A}} \sqcup \mathrm{f}_{\mathrm{B}}$.

Proof. Let $x \in \mathrm{H}$. If $\mathbf{H}_{\mathrm{x}}=\emptyset$, then

$$
\left[\left(f_{A} \diamond f_{B}\right)(x) \cup M\right] \cap N=N \supseteq\left(f_{A}(x) \cup f_{B}(x) \cup M\right) \cap N .
$$

Suppose that $\mathbf{H}_{x} \neq \emptyset$. Let $(u, v) \in \mathbf{H}_{x}$. Then $x \leqslant t$ for some $t \in u \circ v$. Since $f_{A}$ is an $(M, N)$-union soft right hyperideal of $\mathbf{H}$ over $\mathrm{U}$, we obtain that

$$
\begin{aligned}
f_{A}(x) \cap N & =\left(f_{A}(x) \cap N\right) \cap N \\
& \subseteq\left(f_{A}(t) \cup M\right) \cap N \\
& =\left(f_{A}(t) \cap N\right) \cup M \\
& \subseteq\left[\bigcup_{t \in u \circ \mathcal{V}}\left(f_{A}(t) \cap N\right)\right] \cup M \\
& \subseteq\left(f_{A}(u) \cup M\right) \cup M \\
& =f_{A}(u) \cup M .
\end{aligned}
$$

Analogously, we have that $f_{B}(x) \cap N \subseteq f_{B}(v) \cup M$. Therefore, we have that

$$
\left(f_{A}(x) \cup f_{B}(x)\right) \cap N \subseteq\left(f_{A}(u) \cup f_{B}(v)\right) \cup M,
$$

for all $(u, v) \in \mathbf{H}_{x}$. This implies that

$$
\left(f_{A} \diamond f_{B}\right)(x) \cup M=\bigcap_{(u, v) \in \mathbf{H}_{x}}\left(f_{A}(u) \cup f_{B}(v)\right) \cup M \supseteq\left(f_{A}(x) \cup f_{B}(x)\right) \cap N .
$$

Altogether, we obtain that $f_{A} \diamond f_{B} \sqsupseteq[M, N] f_{A} \sqcup f_{B}$.

Theorem 3.11. Let $\mathbf{H}$ be an ordered semihypergroup. Then the following statements are equivalent:

1. $\mathbf{H}$ is regular;

2. $\mathrm{f}_{\mathrm{A}} \diamond \mathrm{f}_{\mathrm{B}}={ }_{[\mathrm{M}, \mathrm{N}]} \mathrm{f}_{\mathrm{A}} \sqcup \mathrm{f}_{\mathrm{B}}$ for any $(\mathrm{M}, \mathrm{N})$-union soft right hyperideal $\mathrm{f}_{\mathrm{A}}$ and any $(\mathrm{M}, \mathrm{N})$-union soft left hyperideal $\mathrm{f}_{\mathrm{B}}$ of $\mathbf{H}$ over $\mathrm{U}$.

Proof. $(1) \Rightarrow(2)$. Let $f_{A}$ and $f_{B}$ be an $[M, N]$-union soft right and an $[M, N]$-union soft left hyperideal of $\mathbf{H}$ over $U$, respectively. Given $a \in H$. Since $H$ is regular, there exists $x \in H$ such that $a \leqslant a \circ x \circ a$. That is, there exists $c \in a \circ x$ such that $a \leqslant c \circ a$, which implies that $\mathbf{H}_{\mathbf{a}} \neq \emptyset$. Then

$$
\begin{aligned}
\left(\left(f_{A} \diamond f_{B}\right)(a) \cup M\right) \cap N & =\left[\bigcap_{(u, v) \in H_{a}}\left(f_{A}(u) \cup f_{B}(v)\right) \cup M\right] \cap N \\
& =\bigcap_{(u, v) \in H_{a}}\left(f_{A}(u) \cup f_{B}(v) \cup M\right) \cap N \\
& \subseteq\left[f_{A}(c) \cup f_{B}(a) \cup M\right] \cap N \\
& =\left(f_{A}(c) \cap N \cap N\right) \cup\left[\left(f_{B}(a) \cup M\right) \cap N\right] \\
& \subseteq\left[\bigcup_{c \in a \circ x} f_{A}(c) \cap N \cap N\right] \cup\left[\left(f_{B}(a) \cup M\right) \cap N\right] \\
& \subseteq\left[\left(f_{A}(a) \cup M\right) \cap N\right] \cup\left[\left(f_{B}(a) \cup M\right) \cap N\right] \\
& =\left[\left(f_{A}(a) \cup f_{B}(a)\right) \cup M\right] \cap N \\
& =\left[\left(f_{A} \cup f_{B}\right)(a) \cup M\right] \cap N .
\end{aligned}
$$

Thus, $f_{A} \sqcup f_{B} \sqsupseteq_{[M, N]} f_{A} \diamond f_{B}$. By Lemma 3.10, we obtain that $f_{A} \diamond f_{B}={ }_{[M, M]} f_{A} \sqcup f_{B}$. 
$(2) \Rightarrow(1)$. Let $R$ and $L$ be a right hyperideal and a left hyperideal of $\mathbf{H}$, respectively. Then, by Lemma 3.6, $\chi_{R}^{c}$ and $\chi_{L}^{c}$ is an $(M, N)$-union soft right hyperideal and an $(M, N)$-union soft left hyperideal of $\mathbf{H}$ over $U$, respectively. Thus, by our presumption, we have that $\chi_{R}^{c} \diamond \chi_{L}^{c}={ }_{[M, N]} \chi_{R}^{c} \cup \chi_{L}^{c}$. Our aim is to show that $\mathbf{H}$ is regular. By Lemma 3.9, it is enough to show that $R \cap L=(R \circ L]$. It is not difficult to see that $\mathrm{R} \cap \mathrm{L} \subseteq(\mathrm{R} \circ \mathrm{L}]$. Thus, we let $\mathrm{a} \in(\mathrm{R} \circ \mathrm{L}]$. Applying Proposition 3.8, we have

$$
\left(\chi_{R}^{c} \sqcup \chi_{L}^{c}\right)(a)={ }_{[M, N]}\left(\chi_{R}^{c} \diamond \chi_{L}^{c}\right)(a)={ }_{[M, N]} \chi_{(R \circ L]}^{c}(a)={ }_{[M, N]} M .
$$

This implies that $\chi_{R}^{c}(a) \subseteq M$ and $\chi_{L}^{c}(a) \subseteq M$. That is, $\chi_{R}^{c}(a)=\emptyset=\chi_{L}^{c}(a)$. It follows that $a \in R$ and $a \in$ L. Thus, $a \in R \cap L$. That is, $(R \circ L] \subseteq R \cap L$. Altogether, we obtain $R \cap L=(R \circ L)$. Therefore, $H$ is regular.

An ordered semihypergroup $\mathbf{H}$ is left (resp., right) weakly regular [6] if for every $a \in H$ there exist $x, y \in H$ such that $a \leqslant x \circ a \circ y \circ a$ (resp., $a \circ x \circ a \circ y)$. Equivalently:

1. $a \in\left((H \circ a)^{2}\right]$ (resp., $\left.a \in\left((a \circ H)^{2}\right]\right)$ for all $a \in H$;

2. $A \subseteq\left((H \circ A)^{2}\right]$ (resp., $\left.A \subseteq\left((A \circ H)^{2}\right]\right)$ for any $A \subseteq H$.

If $\mathbf{H}$ is both a left weakly regular and a right weakly regular, then it is called a weakly regular ordered semihypergroup. It is observed that $\mathbf{H}$ is regular whenever it is commutative and weakly regular.

The following results, we characterize:

1. left (resp., right) weakly regular ordered semihypergroups in terms of $(M, N)$-union soft left (resp., right) hyperideals;

2. weakly regular ordered semihypergroups in terms of $(M, N)$-union soft hyperideals.

Lemma 3.12 ([6, Lemma 3.1]). Let $\mathbf{H}$ be an ordered semihypergroup. Then the following conditions are equivalent:

1. $\mathbf{H}$ is left (resp., right) weakly regular;

2. $\mathrm{L}=(\mathrm{L} \circ \mathrm{L}]$ (resp., $\mathrm{R}=(\mathrm{R} \circ \mathrm{R}])$ for any left hyperideal $\mathrm{L}$ (resp., right hyperideal $\mathrm{R})$ of $\mathbf{H}$;

3. $\mathrm{L}(\mathrm{a})=(\mathrm{L}(\mathrm{a}) \circ \mathrm{L}(\mathrm{a})]($ resp., $\mathrm{R}(\mathrm{a})=(\mathrm{R}(\mathrm{a}) \circ \mathrm{R}(\mathrm{a})])$ for every $\mathrm{a} \in \mathrm{H}$.

Theorem 3.13. Let $\mathbf{H}$ be an ordered semihypergroup. Then the following conditions are equivalent:

1. $\mathbf{H}$ is left weakly regular;

2. $\mathrm{f}_{\mathrm{A}} \diamond \mathrm{f}_{\mathrm{A}}={ }_{[\mathrm{M}, \mathrm{N}]} \mathrm{f}_{\mathrm{A}}$ for any $(\mathrm{M}, \mathrm{N})$-union soft left hyperideal $\mathrm{f}_{\mathrm{A}}$ of $\mathbf{H}$ over $\mathrm{U}$.

Proof. $(1) \Rightarrow(2)$. Let $f_{A}$ be an $(M, N)$-union soft left hyperideal of $\mathbf{H}$ over $U$. Then, by Theorem 3.4, $f_{A} \diamond f_{A} \sqsupseteq_{[M, N]} f_{A}$. On the other hand, let $a \in H$. Since $H$ is left weakly regular, there exist $x, y \in H$ such that $a \leqslant(x \circ a) \circ(y \circ a)$. That is, there exist $u \in x \circ a$ and $v \in y \circ a$ such that $a \leqslant u \circ v$. This implies that $(u, v) \in \mathbf{H}_{a}$, that is $\mathbf{H}_{a} \neq \emptyset$. Thus,

$$
\begin{aligned}
\left(\left(f_{A} \diamond f_{A}\right)(a) \cup M\right) \cap N & =\left[\left(\bigcap_{(u, v) \in H_{a}}\left(f_{A}(u) \cup f_{A}(v)\right)\right) \cup M\right] \cap N \\
& \subseteq\left(f_{A}(u) \cup f_{A}(v) \cup M\right) \cap N \\
& =\left(f_{A}(u) \cup f_{A}(v) \cup M\right) \cap N \cap N \\
& =\left(\left[\left(f_{A}(u) \cap N\right) \cup\left(f_{A}(v) \cap N\right)\right] \cup M\right) \cap N \\
& \subseteq\left(\left[\left(\bigcup_{u \in x \circ a} f_{A}(u) \cap N\right) \cup\left(\bigcup_{v \in x \circ a} f_{A}(v) \cap N\right)\right] \cup M\right) \cap N \\
& \subseteq\left[\left(f_{A}(a) \cup M\right) \cup\left(f_{A}(a) \cup M\right) \cup M\right] \cap N \\
& =\left(f_{A}(a) \cup M\right) \cap N .
\end{aligned}
$$

Therefore, $f_{A} \sqsupseteq_{[M, N]} f_{A} \diamond f_{A}$. Altogether, we have $f_{A} \diamond f_{B}={ }_{[M, N]} f_{A} \sqcup f_{B}$. 
$(2) \Rightarrow(1)$. Let $a \in H$. We show that $L(a)=(L(a) \circ L(a)]$. Let $x \in L(a)$. Then $\chi_{L(a)}^{c}$ is an $(M, N)$-union soft left hyperideal of $\mathbf{H}$ over $\mathrm{U}$. Therefore, by Proposition 3.8,

$$
\chi_{(L(a) \circ L(a)]}^{c}(x)={ }_{[M, N]}\left(\chi_{(L(a)]}^{c} \diamond \chi_{(L(a)]}^{c}\right)(x)={ }_{[M, N]} \chi_{(L(a)]}^{c}(x)=_{[M, N]} M .
$$

This implies that $\chi_{\mathrm{L}(a) \circ L(a)]}^{c}(x) \subseteq M$. That is, $\chi_{\mathrm{L}(a) \circ L(a)]}^{c}(x)=\emptyset$. It follows that $x \in(\mathrm{L}(\mathrm{a}) \circ \mathrm{L}(\mathrm{a})]$. Thus, $\mathrm{L}(\mathrm{a}) \subseteq(\mathrm{L}(\mathrm{a}) \circ \mathrm{L}(\mathrm{a})]$. On the other hand $(\mathrm{L}(\mathrm{a}) \circ \mathrm{L}(\mathrm{a})] \subseteq(\mathrm{H} \circ \mathrm{L}(\mathrm{a})] \subseteq(\mathrm{L}(\mathrm{a})]=\mathrm{L}(\mathrm{a})$. Altogether, we obtain $\mathrm{L}(\mathrm{a})=(\mathrm{L}(\mathrm{a}) \circ \mathrm{L}(\mathrm{a})]$. By Lemma 3.12, $\mathbf{H}$ is left weakly regular.

Similarly, we can prove the following theorem.

Theorem 3.14. Let $\mathbf{H}$ be an ordered semihypergroup. Then the following conditions are equivalent:

1. $\mathbf{H}$ is right weakly regular;

2. $\mathrm{f}_{\mathrm{A}} \diamond \mathrm{f}_{\mathrm{A}}={ }_{[\mathrm{M}, \mathrm{N}]} \mathrm{f}_{\mathrm{A}}$ for any $(\mathrm{M}, \mathrm{N})$-union soft right hyperideal $\mathrm{f}_{\mathrm{A}}$ of $\mathbf{H}$ over $\mathrm{U}$.

Combining Theorem 3.13 and Theorem 3.14, the following result is obtained.

Corollary 3.15. Let $\mathbf{H}$ be an ordered semihypergroup. Then the following conditions are equivalent:

1. $\mathbf{H}$ is weakly regular;

2. $f_{A} \diamond f_{A}=[M, N] f_{A}$ for any $(M, N)$-union soft hyperideal $f_{A}$ of $\mathbf{H}$ over $U$.

An ordered semihypergroup $\mathbf{H}$ is intra-regular [15] if for each element $a \in H$, there exist elements $x, y \in H$ such that $a \leqslant x \circ a \circ a \circ y$. Equivalently:

1. $a \in(H \circ a \circ a \circ H]$ for all $a \in H$;

2. $A \subseteq(H \circ A \circ A \circ H]$ for all $A \subseteq H$.

Lemma 3.16 ([16, Theorem 3.12]). Let $\mathbf{H}$ be an ordered semihypergroup. Then the following conditions are equivalent:

1. $\mathbf{H}$ is intra-regular;

2. $\mathrm{R} \cap \mathrm{L} \subseteq(\mathrm{L} \circ \mathrm{R}]$ for any right hyperideal $\mathrm{R}$ and any left hyperideal $\mathrm{L}$ of $\mathbf{H}$.

Now, we present a characterization of intra-regular ordered semihypergroups as follows.

Theorem 3.17. Let $\mathbf{H}$ be an ordered semihypergroup. Then the following statements are equivalent:

1. $\mathbf{H}$ is intra-regular;

2. $\mathrm{f}_{\mathrm{B}} \sqcup \mathrm{f}_{\mathrm{A}} \sqsupseteq_{[\mathrm{M}, \mathrm{N}]} \mathrm{f}_{\mathrm{B}} \diamond \mathrm{f}_{\mathrm{A}}$ for any $(\mathrm{M}, \mathrm{N})$-union soft right hyperideal $\mathrm{f}_{\mathrm{A}}$ and any $(\mathrm{M}, \mathrm{N})$-union soft left hyperideal $\mathrm{f}_{\mathrm{B}}$ of $\mathbf{H}$ over $\mathrm{U}$.

Proof. $(1) \Rightarrow(2)$. Let $f_{A}$ and $f_{B}$ be an $(M, N)$-union soft right and $(M, N)$-union soft left hyperideal of $\mathbf{H}$ over $U$, respectively. Let $a \in H$. Since $H$ is intra-regular, there exist $x, y \in H$ such that $a \leqslant x \circ a \circ a \circ y$. That is, there exist $u \in x \circ a$ and $v \in a \circ y$ such that $a \leqslant u \circ v$. This implies that $(u, v) \in \mathbf{H}_{a}$, that is, $\mathbf{H} \neq \emptyset$. Thus,

$$
\begin{aligned}
\left(\left(f_{B} \diamond f_{A}\right)(a) \cup M\right) \cap N & =\left[\left(\bigcap_{(u, v) \in \mathbf{H}_{a}}\left(f_{B}(u) \cup f_{A}(v)\right)\right) \cup M\right] \cap N \\
& =\bigcap_{(u, v) \in \mathbf{H}_{a}}\left(f_{A}(u) \cup f_{B}(v) \cup M\right) \cap N \\
& \subseteq\left[f_{A}(u) \cup f_{B}(v) \cup M\right] \cap N \\
& =\left(f_{A}(u) \cap N \cap N\right) \cup\left(f_{B}(v) \cap N \cap N\right) \cup M \\
& \subseteq\left[\bigcup_{u \in x \circ a} f_{A}(u) \cap N \cap N\right] \cup\left[\bigcup_{v \in a \circ y} f_{B}(v) \cap N \cap N\right] \cup M \\
& \subseteq\left[\left(f_{A}(a) \cup M\right) \cap N\right] \cup\left[\left(f_{B}(a) \cup M\right) \cap N\right] \cup M \\
& =\left[\left(f_{A}(a) \cup f_{B}(a)\right) \cup M\right] \cap N .
\end{aligned}
$$

This illustrates that $f_{B} \sqcup f_{A} \sqsupseteq_{[M, N]} f_{B} \diamond f_{A}$. 
$(2) \Rightarrow(1)$. Let $R$ and $L$ be a right hyperideal and a left hyperideal of $\mathbf{H}$ over $\mathrm{U}$, respectively. Then, by Lemma 3.6, $\chi_{R}^{c}$ and $\chi_{L}^{c}$ is an $(M, N)$-union soft right hyperideal and an $(M, N)$-union soft left hyperideal of $\mathbf{H}$ over $\mathrm{U}$, respectively. Thus, by our assumption, we have that $\chi_{R}^{c} \sqcup \chi_{\mathrm{L}}^{\mathrm{c}} \sqsupseteq_{[M, N]} \chi_{\mathrm{L}}^{\mathrm{c}} \diamond \chi_{R}^{\mathrm{c}}$. We show that $\mathbf{H}$ is intra-regular by applying Lemma 3.16. Let $\mathrm{a} \in \mathrm{R} \cap \mathrm{L}$. Thus, by our assumption and Proposition 3.8, we have that

$$
M={ }_{[M, N]}\left(\chi_{R}^{c} \sqcup \chi_{L}^{c}\right)(a) \sqsupseteq_{[M, N]}\left(\chi_{R}^{c} \diamond \chi_{L}^{c}\right)(a)=_{[M, N]} \chi_{(R \circ L]}^{c}(a) .
$$

This implies that $\chi_{(R \circ L]}^{c}(a) \subseteq M$. That is, $\chi_{(R \circ L]}^{c}(a)=\emptyset$. It follows that $a \in(R \circ L]$. Hence, $R \cap L \subseteq(R \circ L]$. Therefore, by Lemma 3.16, $\mathbf{H}$ is intra-regular.

\section{Conclusion}

In this paper, we use the notion of $(M, N)$-union soft left (resp., right) hyperideals in ordered semihypergroups, introduced by Farooq et al. [7], to characterize some regularities of ordered semihypergroups. By our study, it turns out that our results generalize some results investigated by Khan et al. [18]. For our future, we will apply the notions of $(M, N)$-union soft left (resp., right) hyperideals to characterize others regularities of ordered semihypergroups, for example, intra-quasi-regular and completely regular ordered semihypergroups.

\section{Acknowledgment}

The authors are highly grateful to the referees for their valuable comments and suggestions for improving the article.

\section{References}

[1] S. Anvariyeh, S. Mirvakili, B. Davvaz, On Г-hyperideals in Г-semihypergroups, Carpathian J. Math., 26 (2010), 11-23. 1

[2] P. Bonansinga, P. Corsini, On semihypergroup and hypergroup homomorphisms, Boll. Un. Mat. Ital. B (6), 1 (1982), 717-727. 1

[3] P. Corsini, Prolegomena of hypergroup theory, Supplement to Riv. Mat. Pura Appl. Aviani Editore, Tricesimo, (1993). 1

[4] P. Corsini, V. Leoreanu, Applications of hyperstructure theory, Kluwer Academic Publishers, Dordrecht, (2003). 1

[5] B. Davvaz, P. Corsini, T. Changphas, Relationship between ordered semihypergroups and ordered semigroups by using pseudoorder, European J. Combin., 44 (2015), 208-217. 1, 2.1, 2.2, 2.3, 2.4, 2.5

[6] B. Davvaz, A. Khan, M. Farooq, Int-soft structures applied to ordered semihypergroups, Matematiche (Catania), 73 (2018), 235-259. 3, 3.12

[7] M. Farooq, M. Khalaf, A. Khan, More generalizations of union soft hyperideals of ordered semihypergroups, Kragujevac J. Math., 48 (2024), 287-307. 2, 2.7, 2.8, 2.9, 3, 3.2, 3, 3.6, 3.7, 4

[8] Z. Gu, On weakly semiprime segments of ordered semihypergroups, AIMS Math., 6 (2021), 9882-9885. 1

[9] Z. Gu, X. L. Tang, Ordered regular equivalence relations on ordered semihypergroups, J. Algebra, 450 (2016), 384-397. 1

[10] D. Heidari, B. Davvaz, On ordered hyperstructures, Politehn. Univ. Bucharest Sci. Bull. Ser. A Appl. Math. Phys., 73 (2011), 85-96. 1

[11] K. Hila, B. Davvaz, J. Dine, Study on the structure of Г-semihypergroups, Comm. Algebra, 40 (2012), 2932-2948. 1

[12] Y. B. Jun, K. J. Lee, A. Khan, Soft ordered semigroups, MLQ Math. Log. Q., 56 (2010), 42-50. 1

[13] Y. B. Jun, S. Z. Song, G. Muhiuddin, Concave soft sets, critical soft points, and union-soft ideals of ordered semigroups, Sci. World J., 2014 (2014), 12 pages. 1

[14] L. Kamali Ardekani, B. Davvaz, Ordered semihypergroup constructions, Bol. Mat., 25 (2018), 77-99. 1

[15] N. Kehayopulu, Left regular and intra-regular ordered hypersemigroups in terms of semiprime and fuzzy semiprime subsets, Sci. Math. Jpn., 80 (2017), 295-305. 3

[16] N. Kehayopulu, From ordered semigroups to ordered hypersemigroups, Turkish J. Math., 43 (2019), 21-35. 3, 3.9, 3.16

[17] A. Khan, M. Farooq, B. Davvaz, Int-soft interior hyperideals of ordered semihypergroups, Int. J. Anal. Appl., 14 (2017), 193-202. 2

[18] A. Khan, M. Farooq, H. U. Khan, Uni-soft hyperideals of ordered semihypergroups, J. Intell. Fuzzy Systems, 35 (2018), 4557-4571. 2, 2, 4 
[19] A. Khan, M. Farooq, N. Yaqoob, Uni-soft structures applied to ordered $\Gamma$-semihypergroups, Proc. Nat. Acad. Sci. India Sect. A, 90 (2020), 457-465. 1

[20] A. Khan, Y. B. Jun, S. I. Ali Shah, R. Khan, Applications of soft union sets in ordered semigroups via uni-soft quasi-ideals, J. Intell. Fuzzy Systems, 30 (2016), 97-107. 1

[21] A. Khan, R. Khan, Y. B. Jun, Uni-soft structure applied to ordered semigroups, Soft Comput., 21 (2017), 1021-1030. 1

[22] S. Naz, M. Shabir, On soft semihypergroups, J. Intell. Fuzzy Systems, 26 (2014), 2203-2213. 1

[23] T. Mahmood, Some contributions to semihypergroups, PhD thesis, Department of Mathematics, Quaid-i-Azm University, Islamabad, Pakistan, (2011). 1

[24] F. Marty, Sur une generalization de la notion de groupe, In 8th congress Math. Scandinaves, 1934 (1934), 45-49. 1

[25] D. Molodtsov, Soft set theory-first results, Comput. Math. Appl., 37 (1999), 19-31. 1, 2.6, 2

[26] J. Tang, N. Yaqoob, A novel investigation on fuzzy hyperideals in ordered $*$-semihypergroups, Comput. Appl. Math., 40 (2021), 24 pages. 1

[27] N. Yaqoob, P. Corsini, F. Yousafzai, On intra-regular left almost semihypergroups with pure left identity, J. Math., 2013 (2013), 10 pages. 1

[28] N. Yaqoob, I. Cristea, M. Gulistan, S. Nawaz, Left almost polygroups, Ital. J. Pure Appl. Math., 39 (2018), 465-474. 1

[29] P. Yiarayong, B. Davvaz, R. Chinram, On right chain ordered semihypergroups, J. Math. Computer Sci., 24 (2022), 59-72. 1 